MATHEMATICS OF COMPUTATION

Volume 66, Number 220, October 1997, Pages 1663-1687

S 0025-5718(97)00880-6

\title{
ON SOME COMPUTATIONAL PROBLEMS IN FINITE ABELIAN GROUPS
}

\author{
JOHANNES BUCHMANN, MICHAEL J. JACOBSON, JR., AND EDLYN TESKE
}

\begin{abstract}
We present new algorithms for computing orders of elements, discrete logarithms, and structures of finite abelian groups. We estimate the computational complexity and storage requirements, and we explicitly determine the $O$-constants and $\Omega$-constants. We implemented the algorithms for class groups of imaginary quadratic orders and present a selection of our experimental results.

Our algorithms are based on a modification of Shanks' baby-step giantstep strategy, and have the advantage that their computational complexity and storage requirements are relative to the actual order, discrete logarithm, or size of the group, rather than relative to an upper bound on the group order.
\end{abstract}

\section{Introduction}

Let $G$ be a finite abelian group, written multiplicatively, in which we assume that the following are possible:

- for $a, b \in G$ we can compute $c=a * b$

- for $a \in G$ we can compute $a^{-1}$

- for $a, b \in G$ we can test whether $a=b$

We call these the group operations. Note that from every group element $a$ we can determine the neutral element $1=a * a^{-1}$. As an example, we will consider class groups of imaginary quadratic fields. Another example is the group of points on an elliptic curve over a finite field.

For any subset $S$ of $G$, denote by $\langle S\rangle$ the subgroup of $G$ generated by $S$. If $\langle S\rangle=G$, then $S$ is called a generating set of $G$. If $S=\{g\}$, then write $\langle g\rangle$ instead of $\langle S\rangle$.

Three common computational problems in such groups are:

- Given $g \in G$ compute $|\langle g\rangle|$, the order of $g$ in $G$, i.e., the least positive integer $x$ such that $g^{x}=1$.

- Given $g, d \in G$ decide whether $d$ belongs to the cyclic subgroup $\langle g\rangle$ of $G$ generated by $g$. If $d \in\langle g\rangle$, find $\log _{g} d$, the discrete logarithm of $d$ to the base $g$, i.e., the least non-negative integer $x$ such that $g^{x}=d$.

- Given a generating set of $G$ compute the structure of $G$. By computing the structure of $G$ we mean computing positive integers $m_{1}, \ldots, m_{k}$ with $m_{1}>1$,

Received by the editor April 1, 1996 and, in revised form, July 19, 1996.

1991 Mathematics Subject Classification. Primary 11Y16.

The second author was supported by the Natural Sciences and Engineering Research Council of Canada.

The third author was supported by the Deutsche Forschungsgemeinschaft. 
$m_{i} \mid m_{i+1}, 1 \leq i<k$, and an isomorphism $\phi: G \rightarrow \mathbb{Z} / m_{1} \mathbb{Z} \times \cdots \times \mathbb{Z} / m_{k} \mathbb{Z}$. This isomorphism is given in terms of the images of the generators. The integers $m_{i}$ are the uniquely determined invariants of $G$.

In this paper we present improved versions of Shanks' algorithms for solving these problems. As in Shanks' original method [10] (see also [4], [8]), operations in $G$ and table look-ups are used in our algorithms. The table entries are pairs $\left(S^{z}, z\right)$, where $S$ is a set of group elements, $z$ belongs to the set $\mathbb{Z}^{S}$ of all maps $S \longrightarrow \mathbb{Z}$, and $S^{z}=\prod_{g \in S} g^{z(g)}$. When we estimate the complexity of our algorithms, we count the number of group operations, the number of table look-ups, and we determine bounds on the table sizes, i.e., the number of group elements which have to be stored. We ignore the time and space for doing index calculations. If hashing on the group elements is possible, the tables of group elements are hash tables and the time for one table look-up is comparable to the time required for a group operation.

Here are our main results.

Theorem 1.1. There is an algorithm for computing the order $x$ of an element $g \in G$ which executes one inversion and at most

$$
4\lceil\sqrt{x}\rceil+\left\lceil\log \frac{\sqrt{x}}{2}\right\rceil
$$

multiplications in $G$. It uses a table of at most

$$
2\lceil\sqrt{x}\rceil
$$

pairs $(g, r) \in G \times\{1, \ldots, 2\lceil\sqrt{x}\rceil\}$. The total number of table look-ups is bounded by

$$
2\lceil\sqrt{x}\rceil .
$$

Theorem 1.2. There is an algorithm that decides for $g, d \in G, d \neq 1$, whether $d \in\langle g\rangle$ and if so computes $\log _{g} d$. Let

$$
x=\left\{\begin{aligned}
|\langle g\rangle| & \text { if } d \notin\langle g\rangle, \\
\log _{g} d & \text { if } d \in\langle g\rangle .
\end{aligned}\right.
$$

The algorithm executes at most

$$
6\lceil\sqrt{x}\rceil+\lceil\log \sqrt{x}\rceil
$$

multiplications in $G$. It uses a table of at most

$$
2\lceil\sqrt{x}\rceil
$$

pairs $(g, r) \in G \times\{1, \ldots, 2\lceil\sqrt{x}\rceil\}$. The total number of table look-ups is bounded by

$$
4\lceil\sqrt{x}\rceil .
$$

Our algorithm for computing the structure of $G$ may behave differently if the generators are input in different orders. Therefore, the algorithm receives as input a generating sequence, i.e., a finite sequence $S=\left(g_{1}, \ldots, g_{l}\right)$ of group elements such that $\left\{g_{1}, \ldots, g_{l}\right\}$ is a generating set of $G$. Set

$$
G_{j}=\left\langle g_{1}, \ldots, g_{j}\right\rangle, \quad 0 \leq j \leq l,
$$

and

$$
l(S)=\left|\left\{j \in\{1, \ldots, l\}: G_{j-1} \neq G_{j}\right\}\right| .
$$


In other words, if we generate $G$ by using $g_{1}, g_{2}, g_{3}, \ldots, l(S)$ is the number of generators which enlarge the group. Note that $l(S)$ is at least as large as the number of invariants of $G$. We will prove the following result.

Theorem 1.3. There is an algorithm that computes the structure of $G$ from a generating sequence $S$ of $G$ which executes $|S|$ inversions and at most

$$
2^{\frac{l(S)}{2}}(|S|+5) \sqrt{|G|}+4 l(S) \sqrt{|G|}+\log |G|
$$

multiplications in $G$, with $l(S)$ from (2). It uses two tables of at most

$$
2^{\frac{l(S)}{2}} \sqrt{|G|} \quad \text { and } \quad 2^{\frac{l(S)}{2}+1} \sqrt{|G|}
$$

pairs $(g, \vec{q}) \in G \times\{0, \ldots, 2 \sqrt{|G|}\}^{|S|}$. The total number of table look-ups is bounded by

$$
2^{\frac{l(S)}{2}}(|S|+l(S)+2) \sqrt{|G|} .
$$

The upper bound for the complexity of the group structure algorithm is exponential in the number $l(S)$ of generators that are really used to determine the group structure. If that number is fixed, the complexity of the algorithm is $O(|S| \sqrt{|G|})$. On the other hand, our analysis shows that if

$$
G=(\mathbb{Z} / 2 \mathbb{Z})^{l}
$$

for some positive integer $l$, the complexity of our algorithm is $\Omega(|G|)$, where the symbol $\Omega(f(n))$ stands for the set of all functions $g$ such that there exists a constant $M$ with $|g(n)| \geq M|f(n)|$ for all large $n$. This lower bound also holds for Shanks' original algorithm and its variations. Hence, for finite abelian groups with a large number of small invariants our algorithm is not appropriate.

The basic idea of this paper is to use baby-step giant-step algorithms with some initial step-width $v \in 2 \mathbb{N}$ and to double that step-width as long as the result of the computation has not been found. A similar idea has been used in [2] but the results obtained there are weaker than ours. For $v=2$ we obtain the above theorems. If $v$ is chosen such that $L=v^{2}$ is an upper bound on the group order, we obtain Shanks' original algorithms. Our analysis shows that for $v=\sqrt{L}$ the number of multiplications and the table size in the order and discrete logarithm algorithms is $\Omega(\sqrt{L})$. Also, for that choice of $v$ the number of multiplications and the table size in the group structure algorithm is $\Omega\left(2^{l(S) / 2}|S| \sqrt{|L|}\right)$. Thus, if the upper bound $L$ is much larger than the actual order, discrete logarithm or group order, the algorithm wastes a lot of time and space.

We implemented our algorithms for class groups of imaginary quadratic orders using the computer algebra system LiDIA [7]. We present experimental results which yield good choices for the initial step-width $v$ for these groups.

The paper is organized as follows. In Section 2 we describe and analyze the order algorithm. That section also contains the basic idea of the paper. In Section 3 we discuss the algorithm for computing discrete logarithms, and Section 4 is devoted to the group structure algorithm. 


\section{Computing the order of An Element}

Given an element $g \in G$ we wish to compute $x=|\langle g\rangle|$. Our improved algorithm, a modification of Shanks' baby-step giant-step method, is based on the following statement:

Lemma 2.1. Let $v$ be an even positive integer. For every positive integer $x$ there are uniquely determined integers $k, q$ and $r$ with $k \geq 0,\left\lfloor 4^{k-1}\right\rfloor v^{2} \leq 2^{k} v q<4^{k} v^{2}$ and $1 \leq r \leq 2^{k} v$ such that $x=y+r, y=2^{k} v q$.

Proof. Let $x \in \mathbb{N}$. We first show the existence of such $k, q$ and $r$. We choose $k$ such that $\left\lfloor 4^{k-1}\right\rfloor v^{2}<x \leq 4^{k} v^{2}$, and write $x=2^{k} v q+r$ with $1 \leq r \leq 2^{k} v$. Then $\left\lfloor 4^{k-1}\right\rfloor v^{2}-2^{k} v<2^{k} v q \leq 4^{k} v^{2}-1$, which implies that $2^{k} v q<4^{k} v^{2}$. Moreover, if $k=0$, we have $-v<v q$, so $0 \leq v q$. If $k \geq 1$, we have $4^{k-1} v^{2}-2^{k} v<2^{k} v q$; hence $2^{k-1}(v / 2)-1<q$. Since $v / 2$ is integral, this implies that $2^{k-1}(v / 2) \leq q$, so that $4^{k-1} v^{2} \leq 2^{k} v q$.

To show the uniqueness of this representation, let $x=2^{k} v q+r$ with $k, q$ and $r$ as stated above. Then $\left\lfloor 4^{k-1}\right\rfloor v^{2} \leq 2^{k} v q<4^{k} v^{2}$, which implies $q \leq 2^{k} v-1$, so that $x=2^{k} v q+r \leq 2^{k} v\left(2^{k} v-1\right)+2^{k} v=4^{k} v^{2}$. Moreover, we have $\left\lfloor 4^{k-1}\right\rfloor v^{2}<$ $2^{k} v q+r=x$. These inequalities determine $k$ uniquely. The uniqueness of $q$ and $r$ is due to the uniqueness statement for division with remainder.

We explain the method for computing $x=|\langle g\rangle|$. We select an even positive integer $v$ which is used as the initial step-width in the algorithm. Then there is a unique non-negative integer $k$ such that $x$ belongs to the interval

$$
I_{k}=\left\{\left\lfloor 4^{k-1}\right\rfloor v^{2}+1, \ldots, 4^{k} v^{2}\right\} .
$$

We search those intervals for $k=0,1,2, \ldots$ until $x$ is found. By Lemma 2.1, each number in $I_{k}$ can be written as $y+r$ with $y=2^{k} v q$ and $r, q$ as stated in Lemma 2.1. Also, each integer that can be written in this way belongs to the interval $I_{k}$. To check whether $x$ is in $I_{k}$ we test whether $g^{y+r}=1$ with $y=2^{k} v q$ and $r$ and $q$ as stated in Lemma 2.1. This means that we test whether

$$
g^{y}=g^{-r}, \quad 1 \leq r \leq 2^{k} v, \quad y=2^{k} v q, \quad\left\lfloor 4^{k-1}\right\rfloor v^{2} \leq y<4^{k} v^{2} .
$$

For this purpose, we compute the set

$$
R_{k}=\left\{\left(g^{-r}, r\right): 1 \leq r \leq 2^{k} v\right\}
$$

and for all values of $q$ such that $\left\lfloor 4^{k-1}\right\rfloor v^{2} \leq y<4^{k} v^{2}$ we check whether there exists $\left(g^{y}, r\right) \in R_{k}$ for some $r$. If so, $|\langle g\rangle|=y+r$. Otherwise, we increase $k$ by 1 . If $x \leq v$, the set $R_{0}$ contains at least one pair $(1, r)$, and $|\langle g\rangle|=r$ for the smallest such $r$. Therefore, before adding a pair $\left(g^{-r}, r\right), 1 \leq r \leq v$, to $R_{0}$ in the course of the computation of $R_{0}$, we always check whether $g^{-r}=1$, and we break if the answer is "yes", since then $|\langle g\rangle|$ is already found.

The efficiency of the algorithm can be improved if we know a lower bound $B$ of $|\langle g\rangle|$. Writing $C=B-1$, we then work with the set $R_{k}=\left\{\left(g^{-(r+C)}, r\right): 1 \leq r \leq\right.$ $\left.2^{k} v\right\}$, and if we find $\left(g^{y}, r\right) \in R_{k},|\langle g\rangle|=y+r+C$. If no lower bound for $|\langle g\rangle|$ is known, we set $C=0$.

We now present the algorithm. 
Algorithm 2.2.

This algorithm computes the order of the group element $g$ in $G$.

InPuT: $g \in G$, lower bound $C+1$ for $|\langle g\rangle|$, initial step-width $v(v \in 2 \mathbb{N})$ Output: $x=|\langle g\rangle|$

(1) $x=0$

(2) $s=1 ; y=v ; u=v$

(3) $h=g^{-1}$

(4) $a=h^{C} ; b=g^{v} ; c=b$

(5) $R=\emptyset$

(6) while $(x==0)$ do

(7) for $(r=s, s+1 \ldots, u)$ do /* new baby steps $* /$

(8) $a=a * h$$$
\text { if }(s==
$$$$
\text { if }(a==1) \text { then }
$$$$
x=r+C
$$$$
\text { return }(x)
$$

$$
\begin{aligned}
& \text { break while } \\
& \text { else }
\end{aligned}
$$$$
R=R \cup\{(a, r)\}
$$

$$
\mathrm{fi}
$$

$$
\text { else }
$$

$$
R=R \cup\{(a, r)\}
$$

$$
\text { fi }
$$

od
while $\left(x==0\right.$ and $\left.y<u^{2}\right)$ do
/* giant steps $* /$

if (there is a number $r$ such that

$/ * a=g^{-C-r}$;

$b=g^{y} ; c=g^{u} * 1$

/* check if $1 \leq x \leq v * /$

$$
\begin{aligned}
& (b, r) \in R) \text { then } \\
& x=y+r+C \\
& \text { return }(x)
\end{aligned}
$$

$$
\text { else }
$$

$$
\begin{aligned}
& y=y+u \\
& b=b * c
\end{aligned}
$$

$$
\text { fi }
$$$$
\text { od }
$$$$
s=u+1 ; u=2 u
$$$$
c=c^{2}
$$

/* double

od

step-width $*$ /

Theorem 2.3. Let $C=0$. Let $x=|\langle g\rangle|$. For every choice of $v$, Algorithm 2.2 executes one inversion and at most $2\lfloor\log v\rfloor+1$ multiplications in $G$ and requires space for three group elements. On further group multiplications, space required, and table look-ups, we have the following estimates. 
1. If $x \leq v$, Algorithm 2.2 executes $x$ additional multiplications in $G$. It uses a table of $x-1$ pairs $(g, r) \in G \times\{1, \ldots, x-1\}$ and it performs $x$ equality checks.

2. If $\sqrt{x} \leq v<x$, the number $M$ of additional multiplications in $G$ satisfies

$$
v \leq M \leq\lceil\sqrt{x}\rceil+v-2 .
$$

The algorithm uses a table of $v$ pairs $(g, r) \in G \times\{1, \ldots, v\}$, and it performs $v$ equality checks. The total number TL of table look-ups satisfies

$$
1 \leq T L \leq\lceil\sqrt{x}\rceil-1 .
$$

3. If $\sqrt{x}>v$, the number $M$ of additional multiplications in $G$ satisfies

$$
\frac{5}{4}\lceil\sqrt{x}\rceil+\left\lceil\log \frac{\sqrt{x}}{v}\right\rceil-1 \leq M \leq 4\lceil\sqrt{x}\rceil-\frac{v}{2}+\left\lceil\log \frac{\sqrt{x}}{v}\right\rceil-5 .
$$

It performs $v$ equality checks. It uses a table of at least $\lceil\sqrt{x}\rceil$ and at most $2\lceil\sqrt{x}\rceil-2$ pairs $(g, r) \in G \times\{1, \ldots, 2\lceil\sqrt{x}\rceil\}$. The total number $T L$ of table look-ups satisfies

$$
\frac{\lceil\sqrt{x}\rceil}{4} \leq T L \leq 2\lceil\sqrt{x}\rceil-\frac{v}{2}-2 .
$$

Proof. For the initialization, Algorithm 2.2 requires one inversion and at most $2\lfloor\log v\rfloor+1$ multiplications to compute $g^{-1}$ and $b=c=g^{v}$. It must store $g^{-1}, b$ and $c$.

If $x \leq v$, we find $x=|\langle g\rangle|$ during the first iteration of the outer while loop, in the course of the computation of the set $R\left(=R_{0}\right)$. This requires $x$ group multiplications and $x$ equality checks, and the set $R$ contains $x-1$ pairs $\left(g^{-r}, r\right)$.

If $\sqrt{x} \leq v<x$, we also find $x=|\langle g\rangle|$ during the first iteration of the outer while loop. The set $R$ contains $v$ pairs $\left(g^{-r}, r\right)$, which also means that the algorithm must perform $v$ multiplications in $G$ to compute $R$. It performs $v$ equality checks to test whether $g^{-r}=1$. In the iterations of the inner while loop, the algorithm checks whether $\left(g^{q v}, r\right) \in R$ while $v \leq q v \leq x-r(r \in\{1, \ldots, v\})$, i.e., $1 \leq q \leq(x-1) / v$, so we have $1 \leq q<\sqrt{x}$. It computes $g^{q v}$ while $2 v \leq q v \leq x-r$, thus $2 \leq q<\sqrt{x}$. This requires between 1 and $\lceil\sqrt{x}\rceil-1$ table look-ups, and at most $\lceil\sqrt{x}\rceil-2$ group multiplications. Hence, the total number of multiplications in the outer while loop is between $v$ and $v+\lceil\sqrt{x}\rceil-2$, if $\sqrt{x} \leq v<x$.

If $v<\sqrt{x}$, the algorithm performs $k$ additional iterations of the outer while loop, where

$$
4^{k-1} v^{2}<x \leq 4^{k} v^{2}
$$

i.e., $k-1<\log \frac{\sqrt{x}}{v} \leq k$, hence $k=\left\lceil\log \frac{\sqrt{x}}{v}\right\rceil$. After the last iteration, the set $R$ contains $2^{k} v$ pairs $\left(g^{-r}, r\right)$. Thus, to compute $R$ the algorithm performs $2^{k} v$ multiplications in $G$, and it must store a table of $2^{k} v$ pairs. From (3) we see that $2^{k-1} v<\sqrt{x} \leq 2^{k} v$, so

$$
\lceil\sqrt{x}\rceil \leq 2^{k} v \leq 2\lceil\sqrt{x}\rceil-2 .
$$

In the first inner while loop, the algorithm computes $g^{q v}$ for $2 \leq q \leq v$, which requires $v-1$ multiplications. In all of the following inner while loops except the 
last, it computes $g^{q 2^{i} v}$ while

$$
4^{i-1} v^{2}<q 2^{i} v \leq 4^{i} v^{2}
$$

i.e.,

$$
2^{i-1} \frac{v}{2}<q \leq 2^{i} v
$$

where $1 \leq i \leq k-1$. This requires

$$
2^{i} v-2^{i-1} \frac{v}{2}=3 \cdot 2^{i-1} \frac{v}{2}
$$

multiplications in each loop, which can be summed to

$$
\sum_{i=1}^{k-1} 3 \cdot 2^{i-1} \frac{v}{2}=3 \cdot\left(2^{k-1}-1\right) \frac{v}{2}
$$

multiplications. In the last loop, the algorithm computes $g^{q 2^{k} v}$ while

$$
4^{k-1} v^{2}<q 2^{k} v \leq x-r, \quad r \in\left\{1, \ldots, 2^{k} v\right\},
$$

so $2^{k-1} \frac{v}{2}<q \leq \frac{x-1}{2^{k} v}$. Since $\sqrt{x} \leq 2^{k} v$, we have $\frac{x-1}{2^{k} v} \leq \frac{x-1}{\sqrt{x}}<\sqrt{x}$, and this requires at most

$$
\lceil\sqrt{x}\rceil-1-2^{k-1} \frac{v}{2}
$$

multiplications. Thus, the total number of multiplications in the inner while loops is at least

$$
\begin{aligned}
v-1+3 \cdot\left(2^{k-1}-1\right) \frac{v}{2} & =3 \cdot 2^{k-1} \frac{v}{2}-\frac{v}{2}-1 \\
& \stackrel{(4)}{\geq} \frac{3}{4}\lceil\sqrt{x}\rceil-\frac{\sqrt{x}}{2}-1 \geq \frac{\lceil\sqrt{x}\rceil}{4}-1,
\end{aligned}
$$

and at most

$$
\begin{aligned}
v-1+3 \cdot\left(2^{k-1}-1\right) \frac{v}{2}+\lceil\sqrt{x}\rceil-1-2^{k-1} \frac{v}{2} & =2^{k-1} v-\frac{v}{2}+\lceil\sqrt{x}\rceil-2 \\
& \stackrel{(4)}{\leq} 2\lceil\sqrt{x}\rceil-\frac{v}{2}-3,
\end{aligned}
$$

The total number of table look-ups is bounded below by $\lceil\sqrt{x}\rceil / 4$ and above by $2\lceil\sqrt{x}\rceil-\frac{v}{2}-2$, which is the maximum number of iterations of the inner while loops. Finally, note that in $k$ outer while loops the algorithm performs one multiplication to compute $c^{2}$. Together with (4) we get that Algorithm 2.2 performs at least

$$
\left\lceil\log \frac{\sqrt{x}}{v}\right\rceil+\frac{5}{4}\lceil\sqrt{x}\rceil-1
$$

and at most

$$
\left\lceil\log \frac{\sqrt{x}}{v}\right\rceil+4\lceil\sqrt{x}\rceil-\frac{v}{2}-5
$$

additional multiplications in $G$ to find $|\langle g\rangle|$, if $v<\sqrt{x}$.

Remark 2.4. To adapt Theorem 2.3 to the case $C \geq 1$, we just have to replace each $x$ by $x-C$ and add to the total number of group multiplications the multiplications required to compute $a=\left(g^{-1}\right)^{C}$, i.e., at most $2\lfloor\log C\rfloor+1$ multiplications. 
In practice, the most efficient way to handle the set $R$ is by means of a hash table. This is possible as long as the group elements are represented as sequences of integers. Then, each look-up in the table $R$ requires just one computation of a hash value and usually one equality test for group elements.

As we see from Theorem 2.3, the efficiency of Algorithm 2.2 depends largely on the appropriate choice of the initial step-width $v$. As noted by Shanks [10], the optimal choice of $v$ is $v=\sqrt{|\langle g\rangle|}$. This results in about $2 \sqrt{|\langle g\rangle|}$ group multiplications in our algorithm. If $v$ is chosen too large (in comparison with $\sqrt{|\langle g\rangle|}$ ), we waste space and time because the set $R$ is too big. If $v$ is chosen too small, we waste time because of superfluous iterations of the outer while loop.

In order to test our algorithm, we implemented it using the LiDIA system [7] to compute orders of elements in ideal class groups of imaginary quadratic orders. For three discriminants of sizes ten, fifteen, and twenty decimal digits, we computed the orders of the ideal classes of four prime ideals that we knew from previous computations had different orders. In Tables 1, 2, and 3 we show the actual numbers of group multiplications and table look-ups, denoted by $G M$ and $T L$ respectively, that were required to compute the order of each prime ideal class, together with the lower and upper bounds predicted by Theorem 2.3. $\Delta$ denotes the discriminant of the quadratic order and $I_{p}$ denotes the ideal class of which the prime ideal lying over the prime $p$ is the reduced representative. We compute the order of each prime ideal class three times, using a different value of $v$ each time. The simplest version of our algorithm uses $v=2, v=\Delta^{1 / 4}$ is equivalent to Shanks' original algorithm [10], and $v=\Delta^{1 / 4} / 2$ is half-way between the other two and has been shown to yield the best overall run times in our tests. Table 4 gives the run times for these computations on a SPARCstation 20.

TABLE 1. Order algorithm - group multiplications and table look-ups $(v=2)$

\begin{tabular}{|c|r|r|r|r|r|r|r|r|}
\hline & & & \multicolumn{2}{|c|}{ Lower } & \multicolumn{2}{c|}{ Computed } & \multicolumn{2}{c|}{ Upper } \\
\cline { 5 - 8 }$\Delta$ & \multicolumn{1}{|c|}{$\left|\left\langle I_{p}\right\rangle\right|$} & $G M$ & \multicolumn{1}{c|}{$T L$} & \multicolumn{1}{c|}{$G M$} & $T L$ & $G M$ & $T L$ \\
\hline \hline$-4\left(10^{10}+1\right)$ & 5 & 4033 & 85 & 16 & 164 & 94 & 258 & 125 \\
& 3 & 16132 & 166 & 32 & 324 & 189 & 515 & 253 \\
& 13 & 24198 & 202 & 39 & 485 & 221 & 628 & 309 \\
& 7 & 48396 & 282 & 55 & 580 & 316 & 884 & 437 \\
\hline$-4\left(10^{15}+1\right)$ & 7 & 2 & 0 & 0 & 4 & 0 & 5 & 0 \\
& 29 & 42908 & 267 & 52 & 558 & 294 & 836 & 413 \\
& 17 & 128724 & 456 & 89 & 1027 & 506 & 1441 & 715 \\
& 3 & 257448 & 643 & 127 & 1278 & 757 & 2037 & 1013 \\
\hline$-4\left(10^{20}+1\right)$ & 13 & 232024638 & 19054 & 3808 & 38750 & 22352 & 60942 & 30463 \\
& 5 & 464049276 & 26941 & 5385 & 63327 & 30544 & 86179 & 43081 \\
& 37 & 928098552 & 38095 & 7616 & 77489 & 44706 & 121871 & 60927 \\
& 7 & 1856197104 & 53870 & 10771 & 126642 & 61090 & 172348 & 86165 \\
\hline
\end{tabular}

For $\Delta=-4\left(10^{10}+1\right)$, our algorithm using $v=2$ is faster than Shanks' algorithm (i.e. $v=\Delta^{1 / 4}$ ) for $I_{5}$, the class with the smallest order. It is slower for $I_{3}$ and $I_{13}$, even though it executes fewer total group operations, because it performs four 
TABLE 2. Order algorithm - group multiplications and table look-ups $\left(v=\Delta^{1 / 4} / 2\right)$

\begin{tabular}{|c|r|r|r|r|r|r|r|r|}
\hline & & & \multicolumn{2}{|c|}{ Lower } & \multicolumn{2}{c|}{ Computed } & \multicolumn{2}{c|}{ Upper } \\
\cline { 4 - 8 }$\Delta$ & \multicolumn{1}{|c|}{$p$} & \multicolumn{1}{|c|}{$\left|\left\langle I_{p}\right\rangle\right|$} & $G M$ & $T L$ & $G M$ & \multicolumn{1}{c|}{ GL } & \multicolumn{1}{|c|}{$G M$} & $T L$ \\
\hline \hline$-4\left(10^{10}+1\right)$ & 5 & 4033 & 224 & 1 & 251 & 18 & 297 & 63 \\
& 3 & 16132 & 224 & 1 & 305 & 72 & 361 & 127 \\
& 13 & 24198 & 224 & 1 & 341 & 108 & 389 & 155 \\
& 7 & 48396 & 224 & 1 & 449 & 216 & 453 & 219 \\
\hline$-4\left(10^{15}+1\right)$ & 7 & 2 & 0 & 0 & 19 & 0 & 19 & 0 \\
& 29 & 42908 & 3976 & 1 & 4002 & 10 & 4199 & 207 \\
& 17 & 128724 & 3976 & 1 & 4024 & 32 & 4350 & 358 \\
& 3 & 257448 & 3976 & 1 & 4056 & 64 & 4499 & 507 \\
\hline$-4\left(10^{20}+1\right)$ & 13 & 232024638 & 70711 & 1 & 74014 & 3281 & 85965 & 15232 \\
& 5 & 464049276 & 70711 & 1 & 77295 & 6562 & 92274 & 21541 \\
& 37 & 928098552 & 70711 & 1 & 83858 & 13125 & 101197 & 30464 \\
& 7 & 1856197104 & 70711 & 1 & 96983 & 26250 & 113816 & 43083 \\
\hline
\end{tabular}

TABLE 3. Order algorithm - group multiplications and table look-ups $\left(v=\Delta^{1 / 4}\right)$

\begin{tabular}{|c|r|r|r|r|r|r|r|r|}
\hline & & & \multicolumn{2}{|c|}{ Lower } & \multicolumn{2}{c|}{ Computed } & \multicolumn{2}{c|}{ Upper } \\
\cline { 4 - 8 }$\Delta$ & $p$ & $\left|\left\langle I_{p}\right\rangle\right|$ & $G M$ & $T L$ & $G M$ & $T L$ & $G M$ & $T L$ \\
\hline \hline$-4\left(10^{10}+1\right)$ & 5 & 4033 & 448 & 1 & 467 & 9 & 523 & 63 \\
& 3 & 16132 & 448 & 1 & 494 & 36 & 587 & 127 \\
& 13 & 24198 & 448 & 1 & 512 & 54 & 615 & 155 \\
& 7 & 48396 & 448 & 1 & 566 & 108 & 679 & 219 \\
\hline$-4\left(10^{15}+1\right)$ & 7 & 2 & 0 & 0 & 21 & 0 & 21 & 0 \\
& 29 & 42908 & 7953 & 1 & 7977 & 5 & 8178 & 207 \\
& 17 & 128724 & 7953 & 1 & 7988 & 16 & 8329 & 358 \\
$-4\left(10^{20}+1\right)$ & 3 & 257448 & 7953 & 1 & 8004 & 32 & 8478 & 507 \\
& 13 & 232024638 & 141422 & 1 & 143086 & 1640 & 156678 & 15232 \\
& 5 & 464049276 & 141422 & 1 & 144727 & 3281 & 162987 & 21541 \\
& 37 & 928098552 & 141422 & 1 & 148008 & 6562 & 171910 & 30464 \\
& 7 & 1856197104 & 141422 & 1 & 154571 & 13125 & 184529 & 43083 \\
\hline
\end{tabular}

times as many giant steps than Shanks' algorithm, which requires additional table look-ups. In the case of $I_{7}$, the optimal value of $v$ is $v=\sqrt{48396} \approx 220$, and the initial step-width $v=\Delta^{1 / 4} \approx 447$ that is used in Shanks' algorithm is sufficiently accurate that the extra giant steps taken by our algorithm cause it to execute more group operations in total. $v=\Delta^{1 / 4} / 2 \approx 223$ is closer to the optimal value than $v=\Delta^{1 / 4}$, and this choice of $v$ does result in the best overall performance for three of the four ideals.

For $\Delta=-4\left(10^{15}+1\right)$, our algorithm using $v=2$ is the fastest for three of the four prime ideals. The orders of the ideals are sufficiently small that selecting $v=\Delta^{1 / 4}$ or $\Delta^{1 / 4} / 2$ results in too many extra baby steps. For $\Delta=-4\left(10^{20}+1\right)$, 
TABLE 4. Order Algorithm — run times

\begin{tabular}{|c|r|r|r|r|r|}
\hline \multicolumn{1}{|c|}{$\mid r$} & \multicolumn{1}{|c|}{$\left|\left\langle I_{p}\right\rangle\right|$} & $v=2$ & $v=\Delta^{1 / 4} / 2$ & $v=\Delta^{1 / 4}$ \\
\hline \hline$-4\left(10^{10}+1\right)$ & 5 & 4033 & $0.19 \mathrm{sec}$ & $0.16 \mathrm{sec}$ & $0.27 \mathrm{sec}$ \\
& 3 & 16132 & $0.31 \mathrm{sec}$ & $0.22 \mathrm{sec}$ & $0.27 \mathrm{sec}$ \\
& 13 & 24198 & $0.44 \mathrm{sec}$ & $0.30 \mathrm{sec}$ & $0.37 \mathrm{sec}$ \\
$-4\left(10^{15}+1\right)$ & 7 & 48396 & $0.60 \mathrm{sec}$ & $0.44 \mathrm{sec}$ & $0.41 \mathrm{sec}$ \\
& 7 & 2 & $0.13 \mathrm{sec}$ & $0.09 \mathrm{sec}$ & $0.10 \mathrm{sec}$ \\
& 29 & 42908 & $0.86 \mathrm{sec}$ & $2.83 \mathrm{sec}$ & $4.87 \mathrm{sec}$ \\
& 17 & 128724 & $1.20 \mathrm{sec}$ & $2.39 \mathrm{sec}$ & $4.60 \mathrm{sec}$ \\
$-4\left(10^{20}+1\right)$ & 3 & 257448 & $1.74 \mathrm{sec}$ & $1.95 \mathrm{sec}$ & $3.26 \mathrm{sec}$ \\
& 13 & 232024638 & $61.40 \mathrm{sec}$ & $51.80 \mathrm{sec}$ & $92.22 \mathrm{sec}$ \\
& 5 & 464049276 & $81.68 \mathrm{sec}$ & $52.72 \mathrm{sec}$ & $78.71 \mathrm{sec}$ \\
& 37 & 928098552 & $128.06 \mathrm{sec}$ & $78.76 \mathrm{sec}$ & $116.60 \mathrm{sec}$ \\
& 7 & 1856197104 & $172.86 \mathrm{sec}$ & $102.08 \mathrm{sec}$ & $111.14 \mathrm{sec}$ \\
\hline
\end{tabular}

our algorithm using $v=2$ is faster than Shanks' algorithm for one of the four ideals. In this case, $\Delta^{1 / 4}$ is closer to the optimal value of $v$ than for $\Delta=-4\left(10^{15}+1\right)$. Using $v=\Delta^{1 / 4} / 2$, as in the case of $\Delta=-4\left(10^{10}+1\right)$, actually results in the best overall performance.

Our results suggest that Algorithm 2.2 has two main advantages over Shanks' original algorithm. The first and most obvious advantage is that it is faster when the order of the element is much smaller than the order of the group. In these cases, Shanks' algorithm executes too many baby steps, and although our algorithm using $v=2$ will execute some unnecessary giant steps, it will still execute fewer group operations overall. The second advantage is when the upper bound on the order of the group is too large. Shanks' algorithm will execute too many baby steps in this case as well. Our algorithm allows one to select an initial step-width that is much smaller than the estimated order of the group, in the hope of attaining a better approximation of $\sqrt{|\langle g\rangle|}$. Using Shanks' algorithm, an initial step-width that is too small results in far too many giant steps, but our algorithm will detect if the initial step-width is too small and enlarge it if necessary.

\section{COMPuting Discrete LOGARITHMS}

Given $g, d \in G$, we wish to decide whether $d$ belongs to the group $\langle g\rangle$ generated by $g$. If the answer is "yes," we want to compute $x=\log _{g} d$. We use the following modification of Algorithm 2.2 to solve this problem. We compute the order of $g$ in $G$ as in Algorithm 2.2, i.e., we try to find integers $y$ and $r$ such that $g^{y+r}=1$. However, for each $y$, before checking whether $g^{y+r}=1$, we check whether $g^{y+r}=d$. For this, we work with the same set $R$ as in Algorithm 2.2. We first check whether $\left(d^{-1} * g^{y}, r\right) \in R$, with $R$ as in Algorithm 2.2. If this is the case, $\log _{g} d=y+r$. Otherwise, we check whether $\left(g^{y}, r\right) \in R$. As soon as we have found $|\langle g\rangle|$, we know that there is no discrete logarithm of $d$ for base $g$, since $\log _{g} d<|\langle g\rangle|$. Just as in Algorithm 2.2, during the computation of $R_{0}=\left\{\left(g^{-r}, r\right): 1 \leq r \leq v\right\}$ we always check whether $\log _{g} d$ or $|\langle g\rangle|$ is already found before we include a pair $\left(g^{-r}, r\right)$. 


\section{Algorithm 3.1.}

This algorithm computes the discrete logarithm of $d(d \neq 1)$

for base $g$.

InPUT: $d, g \in G(d \neq 1)$, initial step-width $v(v \in 2 \mathbb{N})$

Output: $t=1$ and $x=\log _{g} d$ if $d \in\langle g\rangle$, $t=0$ and $x=|\langle g\rangle|$ if $d \notin\langle g\rangle$

(1) $t=2 ; x=0$

(2) $s=1 ; y=v ; u=v$

(3) $h=g^{-1}$

(4) $a=1 ; b=g^{v} ; c=b$

(5) $R=\emptyset$

(6) $f=d^{-1}$

/*a $a=g^{-r}, b=g^{y}$,

(7) while $(t==2)$ do $c=g^{u} * /$

(8) for $(r=s, s+1$. .,$u$ do new baby steps $* /$

(9) $a=a * h$

$$
a=a * h
$$$$
\text { if }(s==1) \text { then }
$$$$
\text { if }(a==f) \text { then }
$$$$
x=r ; t=1
$$

break while

$$
\begin{aligned}
& \text { else } \\
& \text { if }(a==1) \text { then }
\end{aligned}
$$$$
x=r ; t=0
$$

break while

$$
\text { else }
$$

$$
R=R \cup\{(a, r)\}
$$

fi

fi

$$
\begin{aligned}
& \text { else } \\
& R=R \cup\{(a, r)\}
\end{aligned}
$$

fi

od 


\begin{tabular}{|c|c|c|}
\hline $\begin{array}{l}26) \\
27) \\
28) \\
29) \\
30) \\
31) \\
32) \\
33) \\
34) \\
35) \\
36) \\
37) \\
38) \\
39) \\
40) \\
41) \\
42) \\
43)\end{array}$ & $\begin{array}{l}\text { while }\left(t==2 \text { and } y<u^{2}\right) \text { do } \\
\text { if }(\text { there is a number } r \text { such } \\
\text { that }(f * b, r) \in R) \text { then } \\
x=y+r ; t=1 \\
\text { else } \\
\text { if (there is a number } r \text { such } \\
\text { that }(b, r) \in R) \text { then } \\
\quad x=y+r ; t=0 \\
\text { else } \\
\quad y=y+u \\
\quad b=b * c \\
\quad \mathbf{i} \\
\quad \mathbf{f} \quad \\
\text { od } \begin{array}{l}s=u+1 ; u=2 u \\
c=c^{2}\end{array} \\
\text { od } \\
\text { return }(t, x)\end{array}$ & $\begin{array}{l}/ * \text { giant steps } * / \\
/ * \text { checking for } \\
\text { discrete log. } * /\end{array}$ \\
\hline
\end{tabular}

To discuss the complexity of Algorithm 3.1 we use the same arguments as in Theorem 2.3. We only have to observe that there are twice as many equality checks during the computation of $R_{0}$. Moreover, in each inner while loop, we have one additional multiplication to compute $f * b$ and at most one additional table lookup to check whether $(f * b, r) \in R$. With $x=\log _{g} d$ if $d \in\langle g\rangle$ and $x=|\langle g\rangle|$ if $d \notin\langle g\rangle$, this causes at least 1 additional multiplication and at most $\lceil\sqrt{x}\rceil-1$ additional multiplications and table look-ups if $\sqrt{x} \leq v<x$. If $\sqrt{x}>v$, this causes at least $\lceil\sqrt{x}\rceil / 4-1$ and at most $2\lceil\sqrt{x}\rceil-\frac{v}{2}-2$ additional multiplications and table look-ups. Therefore, we have the following result:

Theorem 3.2. Let $d \neq 1$. Let

$$
x=\left\{\begin{aligned}
|\langle g\rangle| & \text { if } d \notin\langle g\rangle, \\
\log _{g} d & \text { if } d \in\langle g\rangle .
\end{aligned}\right.
$$

For every choice of $v$, Algorithm 3.1 executes two inversions and at most $2\lfloor\log v\rfloor+1$ multiplications in $G$ and requires space for five group elements. On further group multiplications, space required, and table look-ups, we have the following estimates.

1. If $x \leq v$, Algorithm 3.1 executes $x$ further multiplications in $G$. It uses a table of $x-1$ pairs $(g, r) \in G \times\{1, \ldots, x-1\}$, and it performs at most $2 x$ equality checks.

2. If $\sqrt{x} \leq v<x$, the number $M$ of further multiplications in $G$ satisfies

$$
v+1 \leq M \leq 2\lceil\sqrt{x}\rceil+v-3
$$


The algorithm uses a table of $v$ pairs $(g, r) \in G \times\{1, \ldots, v\}$, and it performs $2 v$ equality checks. The total number TL of table look-ups satisfies

$$
1 \leq T L \leq 2\lceil\sqrt{x}\rceil-2
$$

3. If $\sqrt{x}>v$, the number $M$ of further multiplications in $G$ satisfies

$$
\frac{3}{2}\lceil\sqrt{x}\rceil+\left\lceil\log \frac{\sqrt{x}}{v}\right\rceil-2 \leq M \leq 6\lceil\sqrt{x}\rceil-v+\left\lceil\log \frac{\sqrt{x}}{v}\right\rceil-7
$$

It performs at most $2 v$ equality checks. It uses a table of at least $\lceil\sqrt{x}\rceil$ and at most $2\lceil\sqrt{x}\rceil-2$ pairs $(g, r) \in G \times\{1, \ldots, 2\lceil\sqrt{x}\rceil\}$. The total number $T L$ of table look-ups satisfies

$$
\frac{\lceil\sqrt{x}\rceil}{2}-1 \leq T L \leq 4\lceil\sqrt{x}\rceil-v-4
$$

Algorithm 3.1 was implemented using the LiDIA system [7] to compute discrete logarithms in ideal class groups of imaginary quadratic orders. For the same three discriminants used in the previous section, we select the prime ideal class $I_{p}$ with the largest order and use our algorithm to compute $\log _{I_{p}} I_{p}^{x}$ in the class group, for 5 different values of $x$ with varying sizes. Again, we perform the discrete logarithm computation three times using the same three values of $v$ as before. Table 5 shows the number of group multiplications and table look-ups, denoted by $G M$ and $T L$, required for each computation and Table 6 gives the run times for each computation.

TABLE 5. DL algorithm — group multiplications and table look-ups

\begin{tabular}{|c|r|r|r|r|r|r|r|r|}
\hline & \multirow{2}{*}{$\Delta$} & \multicolumn{2}{|c|}{$v=2$} & \multicolumn{2}{c|}{$v=\Delta^{1 / 4} / 2$} & \multicolumn{2}{c|}{$v=\Delta^{1 / 4}$} \\
\cline { 4 - 8 }$\Delta$ & $p$ & $x$ & $G M$ & $T L$ & $G M$ & $T L$ & $G M$ & $T L$ \\
\hline \hline$-4\left(10^{10}+1\right)$ & 7 & 9679 & 411 & 275 & 319 & 85 & 500 & 41 \\
& & 19358 & 668 & 403 & 405 & 171 & 544 & 85 \\
& & 29037 & 744 & 479 & 491 & 257 & 586 & 127 \\
& & 38716 & 820 & 555 & 577 & 343 & 630 & 171 \\
& & 48395 & 896 & 631 & 665 & 431 & 674 & 215 \\
\hline$-4\left(10^{15}+1\right)$ & \multirow{2}{*}{3} & 51491 & 920 & 655 & 4016 & 23 & 7984 & 11 \\
& & 102980 & 1433 & 911 & 4042 & 49 & 7996 & 23 \\
& & 154469 & 1633 & 1111 & 4068 & 75 & 8010 & 37 \\
& & 205958 & 1835 & 1313 & 4094 & 101 & 8022 & 49 \\
& & 257447 & 2035 & 1513 & 4120 & 127 & 8036 & 63 \\
\hline$-4\left(10^{20}+1\right)$ & 7 & 371239423 & 88207 & 55423 & 81233 & 10499 & 146696 & 5249 \\
& & 742478843 & 110865 & 78081 & 91733 & 20999 & 151946 & 10499 \\
& & 1113718263 & 165072 & 99519 & 102233 & 31499 & 157196 & 15749 \\
& & 1484957683 & 176402 & 110849 & 112733 & 41999 & 162446 & 20999 \\
& & 1856197103 & 187732 & 122179 & 123233 & 52499 & 167696 & 26249 \\
\hline
\end{tabular}


TABLE 6. DL algorithm — run times

\begin{tabular}{|c|c|c|c|c|c|}
\hline$\Delta$ & $p$ & $x$ & $v=2$ & $v=\Delta^{1 / 4} / 2$ & $v=\Delta^{1 / 4}$ \\
\hline \multirow[t]{5}{*}{$-4\left(10^{10}+1\right.$} & \multirow[t]{5}{*}{7} & 9679 & $0.34 \mathrm{sec}$ & $0.20 \mathrm{sec}$ & $0.24 \mathrm{sec}$ \\
\hline & & 19358 & 0.53 & $0.28 \mathrm{sec}$ & $0.28 \mathrm{sec}$ \\
\hline & & 29037 & 0.58 & $0.36 \mathrm{sec}$ & $0.32 \mathrm{sec}$ \\
\hline & & 38716 & 0.66 & $0.45 \mathrm{sec}$ & $0.37 \mathrm{sec}$ \\
\hline & & 48395 & $0.56 \mathrm{sec}$ & $0.40 \mathrm{sec}$ & $0.34 \mathrm{sec}$ \\
\hline \multirow[t]{5}{*}{$-4\left(10^{15}+1\right)$} & \multirow[t]{5}{*}{3} & 51491 & 1.28 & $1.55 \mathrm{sec}$ & $3.13 \mathrm{sec}$ \\
\hline & & 102980 & $1.80 \mathrm{sec}$ & $1.51 \mathrm{sec}$ & $3.09 \mathrm{sec}$ \\
\hline & & 154469 & $2.05 \mathrm{sec}$ & $1.67 \mathrm{sec}$ & $2.73 \mathrm{sec}$ \\
\hline & & 205958 & $2.20 \mathrm{sec}$ & $1.44 \mathrm{sec}$ & $2.63 \mathrm{sec}$ \\
\hline & & 257447 & $1.53 \mathrm{sec}$ & $1.40 \mathrm{sec}$ & $3.22 \mathrm{sec}$ \\
\hline \multirow[t]{5}{*}{$-4\left(10^{20}+1\right)$} & \multirow[t]{5}{*}{7} & 371239423 & $130.04 \mathrm{sec}$ & $55.17 \mathrm{sec}$ & $80.03 \mathrm{sec}$ \\
\hline & & 742478843 & $179.09 \mathrm{sec}$ & $80.61 \mathrm{sec}$ & $90.70 \mathrm{sec}$ \\
\hline & & 1113718263 & $238.34 \mathrm{sec}$ & $101.31 \mathrm{sec}$ & $106.19 \mathrm{sec}$ \\
\hline & & 1484957683 & $264.40 \mathrm{sec}$ & $127.83 \mathrm{sec}$ & $113.06 \mathrm{sec}$ \\
\hline & & 1856197103 & $188.05 \mathrm{sec}$ & $105.26 \mathrm{sec}$ & $106.34 \mathrm{sec}$ \\
\hline
\end{tabular}

In the DL Algorithm, the advantages of our method in comparison with Shanks' method become even clearer. This is due to the fact that for $\log _{g} d$ all values between 0 and $|\langle g\rangle|$ are possible, and they are equally probable (this is, however, not the case in cryptographic circumstances). Assuming that $|\langle g\rangle|$ is not known a priori, Shanks' original algorithm still uses the upper bound of the group order, i.e., $v=\Delta^{1 / 4}$, which causes the algorithm to perform far too many baby steps.

For $\Delta=-4\left(10^{10}+1\right)$ and the prime ideal lying over 7 , in which case the bound $v=\Delta^{1 / 4}$ is quite accurate, Shanks' algorithm is faster than our algorithm using $v=$ $\Delta^{1 / 4} / 2$ for about half of the computed logarithms in our experiments. For all DL's smaller than half of the order of $I_{7}$, our algorithm using $v=\Delta^{1 / 4} / 2$ works better. The comparatively bad results for our algorithm using $v=2$ are due to the fact that in the DL Algorithm each giant step causes two group multiplications and two table look-ups, so that too many giant steps cause twice as much unnecessary work as in the Order Algorithm. Note that computing discrete logarithms of elements generated by $I_{7}$ represents the best case for Shanks' algorithm and the worst case for our algorithm because $I_{7}$ is the largest prime ideal.

For $\Delta=-4\left(10^{15}+1\right)$ and $\Delta=-4\left(10^{20}+1\right)$, where the upper bounds of the group order are much larger than the orders of the largest prime ideals, our algorithm using $v=\Delta^{1 / 4} / 2$ works better for almost all possible logarithms.

Thus, our experiments lead to similar conclusions to those related to Algorithm 2.2, namely that our algorithm works better when $\log _{g} d$ is small compared to $|\langle g\rangle|$ and when the upper bound on the order of the group is too large.

\section{Computing the structure of a subgroup}

Given a generating system, i.e., a finite sequence $S=\left(g_{1}, \ldots, g_{l}\right)$, of a finite abelian group $G$ that is given as described in the introduction, we want to find the 
structure of $G$. By this we mean finding positive integers $m_{1}, \ldots, m_{k}$ with $m_{1}>1$, $m_{j} \mid m_{j+1}, 1 \leq j<k$, and an isomorphism

$$
\phi: G \longrightarrow \mathbb{Z} / m_{1} \mathbb{Z} \times \cdots \times \mathbb{Z} / m_{k} \mathbb{Z}
$$

This isomorphism will be given in terms of the images of the elements of the generating system $S$. The integers $m_{i}$ are the invariants of $G$.

We describe our method. For $\vec{z}=\left(z_{1}, \ldots, z_{l}\right) \in \mathbb{Z}^{l}$ we write

$$
S^{\vec{z}}=\prod_{i=1}^{l} g_{i}^{z_{i}} .
$$

A relation on $S$ is a vector $\vec{z} \in \mathbb{Z}^{l}$ such that $S^{\vec{z}}=1$. The set $L(S)$ of all relations on $S$ is a lattice in $\mathbb{Z}^{l}$ of dimension $l$, since it is the kernel of the surjective homomorphism

$$
\mathbb{Z}^{l} \longrightarrow G, \quad \vec{z} \mapsto S^{\vec{z}}
$$

Our approach will be to compute a basis $B=\left(\vec{b}_{1}, \ldots, \vec{b}_{l}\right)$ of $L(S)$. That basis is identified with a matrix of column vectors $\vec{b}_{j}=\left(b_{1 j}, \ldots, b_{l j}\right)^{t}$. Then the order of $G$ is $|\operatorname{det} B|$. Using standard techniques, e.g. [6], [4], we compute the Smith normal form $N$ of $B$ and a matrix $U \in \mathbb{Z}^{l \times l}$ with $\operatorname{det} U \not \equiv 0 \bmod |G|$ such that there exists $V \in \mathbb{Z}^{l \times l}$ with $\operatorname{det} V \not \equiv 0 \bmod |G|$ and $N \equiv U B V \bmod |G|$. This means that we do not need to find two unimodular transformations for the Smith normal form but only a left unimodular transformation $\bmod G$. Therefore, modular techniques for finding the Smith normal form can be applied.

Let $N$ be the diagonal matrix $\operatorname{diag}\left(1, \ldots, 1, m_{1}, \ldots, m_{k}\right)$ where $m_{1}>1$. Then $m_{1}, \ldots, m_{k}$ are the invariants of $G$. To define the map (5) delete in $U$ all but the last $k$ rows. Let $\vec{u}_{1}, \ldots, \vec{u}_{l}$ be the column vectors of that new matrix, $\vec{u}_{j}=$ $\left(u_{1 j}, \ldots, u_{k j}\right)^{t}$. Set

$$
\phi\left(g_{j}\right)=\left(u_{1 j} \bmod m_{1}, \ldots, u_{k j} \bmod m_{k}\right), \quad 1 \leq j \leq l .
$$

Then $\phi$ is the isomorphism we were looking for.

We describe the computation of the basis $B$ of the relation lattice $L(S)$. The matrix $B$ will be an upper triangular matrix with positive entries on the diagonal.

Suppose that we have computed $\vec{b}_{1}, \ldots, \vec{b}_{j-1}$. We describe the computation of $\vec{b}_{j}$. The diagonal entry $b_{j j}$ is the smallest positive integer such that $g_{j} b_{j j}$ belongs to the subgroup of $G$ generated by $\left\{g_{1}, \ldots, g_{j-1}\right\}$. The problem of finding $\vec{b}_{j}$ is therefore similar to a discrete logarithm problem. Indeed, our solution is an extension of Algorithm 3.1. Instead of a single initial step-width $v$ we now use a vector $\vec{v}=\left(v_{1}, \ldots, v_{l}\right) \in 2 \mathbb{N}^{l}$ of step-widths. Since there is a basis $B$ with $0 \leq b_{i j}<b_{i i}$ for $1 \leq i<j \leq l$ we can write

$$
b_{i j}=q_{i} B_{i}+r_{i}
$$


where $B_{i}=\left\lceil\sqrt{b_{i i}}\right\rceil, 0 \leq q_{i}<B_{i}$, and $0 \leq r_{i}<B_{i}$. We also write

$$
b_{j j}=y+r_{j}
$$

where $y=2^{k} v_{j} q$ and $1 \leq r_{j} \leq 2^{k} v_{j}$ with $k$ and $q$ as in Lemma 2.1. Set

$$
S_{i}=\left(g_{1}, \ldots, g_{i}\right), \quad 1 \leq i \leq l .
$$

As in Algorithms 2.2 and 3.1 we proceed to find the smallest value of $y$ such that

$$
S_{j-1} \vec{q} \vec{B} g_{j}^{y}=S_{j}^{-\vec{r}}
$$

where

$$
\vec{q}=\left(q_{1}, \ldots, q_{j-1}\right), \quad 0 \leq q_{i}<B_{i}, q_{i} B_{i}<b_{i i}, 1 \leq i<j,
$$

$\vec{B}=\left(B_{1}, \ldots, B_{j-1}\right), \vec{q} \vec{B}$ denotes the componentwise multiplication, and

$$
\vec{r}=\left(r_{1}, \ldots, r_{j}\right), \quad 0 \leq r_{i}<B_{i}, 1 \leq i<j, \text { and } 1 \leq r_{j} \leq 2^{k} v_{j} .
$$

Once $y$ is found we have

$$
\vec{b}_{j}=\vec{q} \vec{B} \circ(y) \circ \underbrace{(0, \ldots, 0)}_{l-j}+\vec{r} \circ \underbrace{(0, \ldots, 0)}_{l-j}
$$

where $\circ$ denotes the concatenation of vectors.

To be able to check quickly whether (6) holds for a given $y$ we use the sets

$$
Q=\left\{\left(S_{j-1} \vec{q} \vec{B}, \vec{q}\right): \vec{q} \text { as in }(7)\right\}
$$

and

$$
R=\left\{\left(S_{j} \vec{r}, \vec{r}\right): \vec{r} \text { as in }(8)\right\} .
$$

Just as in the order algorithm and the discrete logarithm algorithm we check whether $b_{j j} \leq v_{j}$ already during the first computation of $R$, i.e., when $k=0$ in (8). For this we use the set

$$
R^{\prime}=\left\{\left(S_{j-1}^{\vec{r}}: 0 \leq r_{i}<B_{i}, 1 \leq i<j\right\}\right.
$$

Moreover, before computing any element of $R$ we check if $b_{j j}=1$. This is done separately, because in many cases the algorithm will compute the entire group structure with only a few generators, and all the others can be handled by this special case. For this check we use the set $Q$ instead of $R^{\prime}$, since in general $Q$ is considerably smaller than $R^{\prime}$.

Here is the algorithm which determines the HNF-basis $B$ for the relation lattice $L(S)$. 


\section{Algorithm 4.1.}

This algorithm computes the HNF-basis for the lattice of

relations on a generating system for a finite abelian group.

INPUT: A generating system $S=\left(g_{1}, \ldots, g_{l}\right)$ of $G$, initial step-width $\vec{v}=\left(v_{1}, \ldots, v_{l}\right) \in 2 \mathbb{N}^{l}$.

Output: A basis $\mathcal{B}=\left(\vec{b}_{1}, \ldots, \vec{b}_{l}\right)$ of the lattice of relations on $S$ in upper triangular form.

(1) $\quad R^{\prime}=\{(1,())\}, Q=\{(1,())\}, \quad \quad / *$ initialization $* /$ $\vec{B}=()$

(2) for $(j=1, \ldots, l)$ do

(3) $s=1, y=v_{j}, u=v_{j}$

(4) $h=g_{j}{ }^{-1}$

(5) $\quad a=1, b=g_{j}{ }^{v_{j}}, c=b$

(6) $\quad R=\emptyset$

(7) for $($ all $(e, \vec{q}) \in Q)$ do

(8) $\quad d=e * g_{j}$ (9) if ( there is $\vec{r}$ such that $(d, \vec{r}) \in R^{\prime} \quad$ subgroup $*$ /

/* $a=g_{j}{ }^{-i}, b=g_{j}{ }^{y}$, $c=g_{j}{ }^{u} * /$

/* check whether $g_{j}$ is

/* new baby steps $* /$

/* check whether $1<b_{j j} \leq v_{j} * /$

$$
\begin{aligned}
& \text { od } \\
& \text { while }\left(b_{j j}==0\right) \text { do } \\
& \text { for }(i=s, \ldots, u) \text { do } \\
& a=a * h \\
& \begin{array}{rll}
\text { if }(s==1 \text { and } i>1) & \text { then } & / * \text { check whether } \\
\text { for }\left(\text { all }(d, \vec{r}) \in R^{\prime}\right) & \text { do } & 1<b_{j j} \leq v_{j} * /
\end{array} \\
& e=d * a \\
& \text { if ( there is } \vec{q} \text { such that }(e, \vec{q}) \in Q \\
& \text { and } q_{i}+r_{i}<b_{i i} \forall i=1, \ldots, j-1 \\
& \text { ) then } \\
& \vec{b}_{j}=\vec{q} \vec{B} \circ(0) \circ(0, \ldots, 0)+\vec{r} \circ \\
& (i) \circ(0, \ldots, 0) \\
& \text { break while } \\
& \text { else } \\
& R=R \cup\{(e, \vec{r} \circ(i))\} \\
& \text { fi } \\
& \text { od } \\
& \text { else } \\
& R=R \cup\{(d * a, \vec{r} \circ(i)):(d, \vec{r}) \in \\
& \text { fi } \\
& \left.R^{\prime}\right\} \\
& \text { od }
\end{aligned}
$$




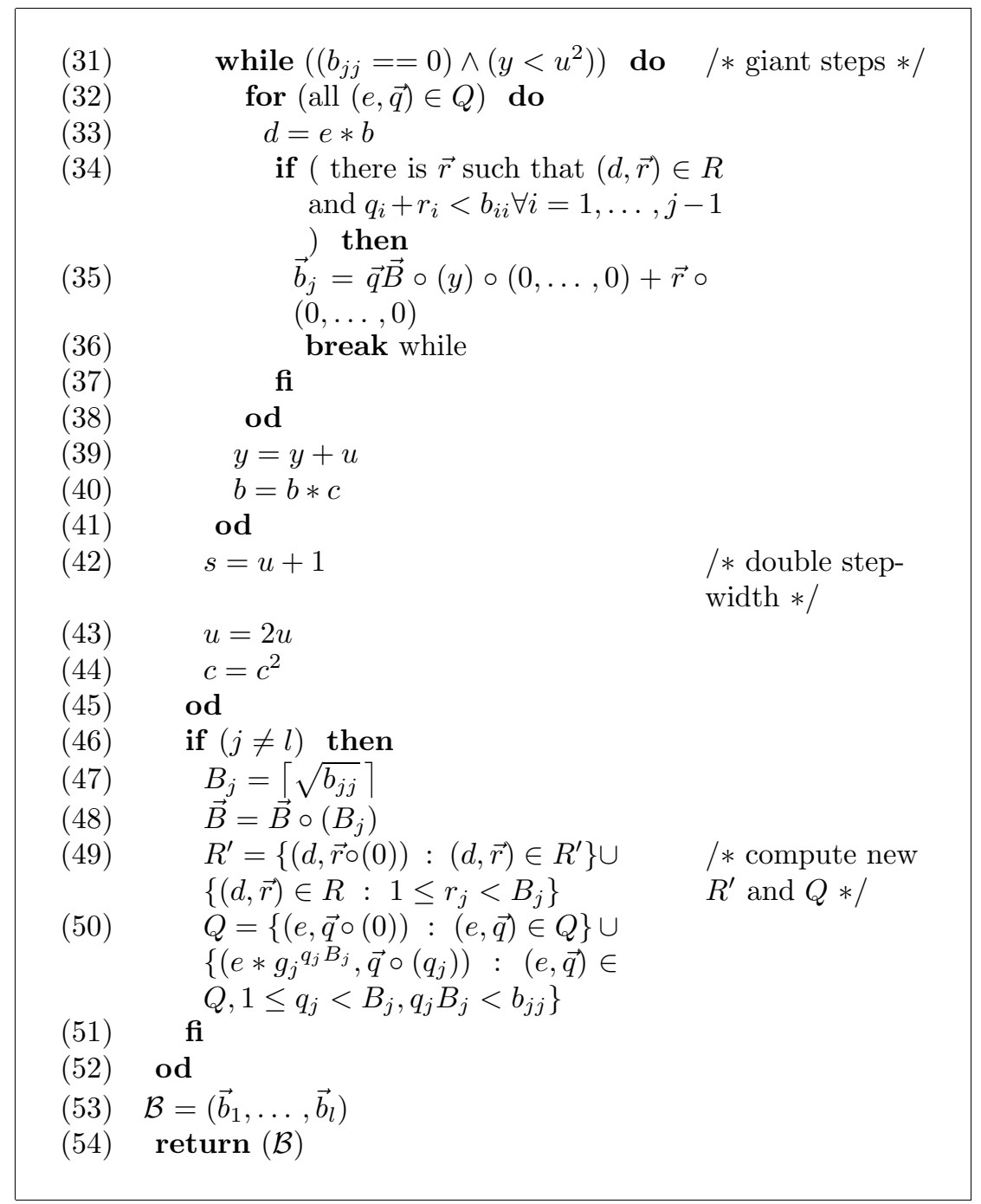

We implemented our algorithm using the LiDIA system [7] to compute, once again, in ideal class groups of imaginary quadratic orders. During the course of the implementation, we found that the sets $R^{\prime}, R$, and $Q$ are most efficiently stored as indexed hash tables, since the algorithm requires traversing the tables in addition to fast searching. Using this data structure also allows one to maintain $R^{\prime}$ and $R$ in one table. In the interest of saving storage, the exponent vectors in these tables are encoded into single integers.

For simplicity, we analyze the complexity of Algorithm 4.1 only with initial stepwidth $\vec{v}=(2, \ldots, 2)$. We need the following lemma.

Lemma 4.2. Let $m_{1}, \ldots, m_{l} \in \mathbb{N}$ and $e=\left|\left\{m_{j} \mid m_{j}>1\right\}\right|$. Then we have

$$
\prod_{j=1}^{l}\left\lceil\sqrt{m_{j}}\right\rceil \leq 2^{\frac{e}{2}} \cdot \prod_{j=1}^{l} \sqrt{m_{j}} .
$$

Equality holds if and only if $m_{j}=2$ for all $m_{j}$ with $m_{j}>1$. 
Proof. Let $m \in \mathbb{N}$. If $m=1,\lceil\sqrt{m}\rceil=\sqrt{m}$. If $m=2,\lceil\sqrt{m}\rceil=\sqrt{2} \sqrt{m}$. If $3 \leq m \leq 5,\lceil\sqrt{m}\rceil<\sqrt{2} \sqrt{m}$. If $m \geq 6,1<\sqrt{6}(\sqrt{2}-1) \leq \sqrt{m}(\sqrt{2}-1)$, thus $\lceil\sqrt{m}\rceil<\sqrt{m}+1<\sqrt{2} \sqrt{m}$. Hence, $\lceil\sqrt{m}\rceil \leq \sqrt{2} \sqrt{m}$ for all $m \geq 2$, with equality if and only if $m=2$. From this our claim follows.

Theorem 4.3. Let $\vec{v}=\left(v_{1}, \ldots, v_{l}\right)$ with $v_{1}=\cdots=v_{l}=2$. Algorithm 4.1 executes $l$ inversions and at most

$$
2^{\frac{l(S)}{2}}(l+5) \sqrt{|G|}+4 l(S) \sqrt{|G|}+\log |G|
$$

multiplications in $G$, where $l$ denotes the number of generators and $l(S)$ denotes the number of the $b_{j j}$ that are larger than 1 . It uses two tables of at most

$$
2^{\frac{l(S)}{2}} \sqrt{|G|} \text { and } 2^{\frac{l(S)}{2}+1} \sqrt{|G|}
$$

pairs $(e, \vec{q}) \in G \times\{0, \ldots,|G|\}^{l}$. The total number of table look-ups is bounded by $2^{\frac{l(S)}{2}}(l+l(S)+2) \sqrt{|G|}$.

Proof. Algorithm 4.1 performs $l$ outer loops to compute the column vectors $\vec{b}_{1}, \ldots$, $\vec{b}_{l}$. We first estimate the sizes of the sets $Q, R$ and $R^{\prime}$ for each of these outer loops, and how many group operations are needed to build up these sets. Let $Q_{j}, R_{j}$ and $R_{j}^{\prime}$ denote the sets $Q, R$ and $R^{\prime}$ after the $j$-th loop. We have

$$
\left|Q_{0}\right|=1 \quad \text { and } \quad\left|Q_{j}\right| \leq\left|Q_{j-1}\right| \cdot B_{j},
$$

where $B_{j}=\left\lceil\sqrt{b_{j j}}\right\rceil$. Thus $\left|Q_{j}\right| \leq \prod_{i=1}^{j} B_{i}$. Writing

$$
T=\prod_{i=1}^{l} B_{i},
$$

we get that $\left|Q_{j}\right| \leq T$ for all $j$. It takes at most

$$
\left|Q_{j-1}\right| \cdot\left(B_{j}-1\right) \leq\left(B_{j}-1\right) \cdot \prod_{i=1}^{j-1} B_{i}
$$

multiplications to compute $Q_{j}$ given $Q_{j-1}$. The maximum number $M_{Q}$ of multiplications to compute all the sets $Q_{1}, \ldots, Q_{l-1}$ can be estimated as follows:

$$
\begin{aligned}
M_{Q} \leq \sum_{j=1}^{l-1}\left(B_{j}-1\right) \prod_{i=1}^{j-1} B_{i} & =\sum_{j=1}^{l-1}\left(\prod_{i=1}^{j} B_{i}-\prod_{i=1}^{j-1} B_{i}\right) \\
& =\prod_{j=1}^{l-1} B_{j}-1 \leq T-1 .
\end{aligned}
$$

In considering $R_{j}$, we use the arguments of the proof of Theorem 2.3, with initial step-width $v=2$. At the end of the $j$-th loop, the set $R\left(=R_{j}\right)$ consists of (at most) $p_{j}\left|R_{j-1}^{\prime}\right|$ pairs $\left(d * g_{j}{ }^{i}, \vec{r}\right)$ with $d \in R_{j-1}^{\prime}$ and $1 \leq i \leq p_{j}$, where $p_{j}=0$ if $b_{j j}=1, p_{j}=1$ if $b_{j j}=2, p_{j}=2$ if $2<b_{j j} \leq 4$, and $p_{j} \leq 2\left\lceil\sqrt{b_{j j}}\right\rceil-2$ if $2<\sqrt{b_{j j}}$. So we have $p_{j} \leq 2 B_{j}-2$ in all cases. Hence

$$
\left|R_{j}\right| \leq\left(2 B_{j}-2\right)\left|R_{j-1}^{\prime}\right| .
$$

Moreover, the algorithm performs at most $2 B_{j}-2$ multiplications $a=a * h$ (step (16)), so that the maximum number of multiplications needed to compute $R_{j}$ given 
$R_{j-1}^{\prime}$ can be estimated by $\left(2 B_{j}-2\right)\left|R_{j-1}^{\prime}\right|+2 B_{j}-2$. From the way in which $\left|R^{\prime}\right|$ is built up, we see that

$$
\left|R_{j}^{\prime}\right|=\left|R_{j-1}^{\prime}\right|+\left(B_{j}-1\right)\left|R_{j-1}^{\prime}\right|=B_{j}\left|R_{j-1}^{\prime}\right|,
$$

where $\left|R_{0}^{\prime}\right|=1$. Thus,

$$
\left|R_{j}^{\prime}\right|=\prod_{i=1}^{j} B_{i}, \quad j=1, \ldots, l-1,
$$

and

$$
\left|R_{j}\right| \leq 2\left(B_{j}-1\right) \prod_{i=1}^{j-1} B_{i}, \quad j=1, \ldots, l,
$$

so that we always have $\left|R^{\prime}\right| \leq T$ and $|R| \leq 2 T$. Therefore, the algorithm requires space for at most $4 T$ pairs $(e, \vec{q}) \in G \times\{0, \ldots,|G|\}^{l}$ to store $Q, R$ and $R^{\prime}$. Let

$$
\begin{aligned}
E & =\left\{g_{j}: j \in\{1, \ldots, l\}, b_{j j} \geq 2\right\} \\
& =\left\{g_{j}: j \in\{1, \ldots, l\}, G_{j-1} \neq G_{j}\right\},
\end{aligned}
$$

where $G_{j}=\left\langle g_{1}, \ldots, g_{j}\right\rangle, 0 \leq j \leq l$. Let $l(S)=|E|$. Then the maximum number $M_{R}$ of multiplications required to compute all the sets $R_{j}$ satisfies

$$
\begin{aligned}
M_{R} & \leq 2\left[\sum_{j=1}^{l}\left(B_{j}-1\right) \prod_{i=1}^{j-1} B_{i}+\sum_{j=1}^{l}\left(B_{j}-1\right)\right] \\
& =2\left[\prod_{j=1}^{l} B_{j}-1+\sum_{j=1}^{l}\left(B_{j}-1\right)\right] \\
& \leq 2 T-2+2 \sum_{\substack{j=1 \\
g_{j} \in E}}^{l} b_{j j} \leq 2 T-2+2 l(S) \cdot \sqrt{|G|} .
\end{aligned}
$$

Next, let us consider the remaining group multiplications and table look-ups in the $l$ outer loops. It takes $l$ inversions and $l$ multiplications to compute $g_{j}{ }^{-1}$ and $g_{j}{ }^{2}$ at the beginning of each outer loop. To check whether $b_{j j}=1$, i.e., whether $g_{j}$ is contained in the subgroup generated by $g_{1}, \ldots, g_{j-1}$, the algorithm checks for at most $|Q|$ elements $e$ whether $\left(e * g_{j}, \vec{r}\right) \in R^{\prime}$ for some $\vec{r}$. In total, this requires at most

$$
l \cdot|Q| \leq l \cdot T
$$

multiplications and table look-ups. We only have iterations of the outer while loops $((14)-(45))$ if $g_{j} \in E$. To check whether $1<b_{j j} \leq 2\left(=v_{j}\right)$, the algorithm checks for at most $\left|R^{\prime}\right|$ elements $d$ whether $\left(d * g_{j}{ }^{-b_{j j}}, \vec{q}\right) \in Q$ for some $\vec{q}$. This requires at most $\left|R^{\prime}\right| \leq\left(b_{j j}-1\right) T=T$ table look-ups for each $g_{j} \in E$, so altogether at most

$$
l(S) \cdot T
$$

table look-ups (the multiplications have already been considered above). We only have further computations in the $j$-th outer loop if $2<b_{j j}$. In this case, we have to distinguish between the cases $\sqrt{b_{j j}} \leq 2$ and $2<\sqrt{b_{j j}}$. If $\sqrt{b_{j j}} \leq 2$, i.e., $b_{j j}=3$ or 4 , then $b_{j j}$ is found during the first iteration of the outer while loop. We conclude from the proof of Theorem 2.3 that it takes at most $\left(B_{j}-1\right)\left|Q_{j-1}\right|=\left|Q_{j-1}\right|$ table 
look-ups and multiplications to check whether some element $(e * b, \vec{r})$ is in $R$ (steps $(33) /(34))$, and it requires at most $B_{j}-2(=0)$ multiplications $b=b * c$ (step (40)), plus one additional multiplication in step (44). If $2<\sqrt{b_{j j}}$, we have in total

$$
k_{j}=\left\lceil\log \frac{b_{j j}}{2}\right\rceil+1=\left\lceil\log b_{j j}\right\rceil
$$

iterations of the outer while loop, which means in all $\left\lceil\log b_{j j}\right\rceil$ multiplications for squaring $c$ in step (44). We use the results of the proof of Theorem 2.3 to see that in the inner while loops $((31)-(41))$, the algorithm performs at most $\left(2 B_{j}-3\right)\left|Q_{j-1}\right|$ table look-ups and multiplications in steps $(33) /(34)$ and at most $2 B_{j}-4$ multiplications in step (40). Combining the results of the cases $\sqrt{b_{j j}} \leq 2$ and $2 \leq \sqrt{b_{j j}}$, we get that for each $g_{j} \in E$ the algorithm performs at most

$$
\left(2 B_{j}-3\right)\left|Q_{j-1}\right|
$$

table look-ups in the inner while loops and at most

$$
\left(2 B_{j}-3\right)\left|Q_{j-1}\right|+2 B_{j}-4+\left\lceil\log b_{j j}\right\rceil
$$

group multiplications in the inner while loops and step (44). The maximum total number of table look-ups in the inner while loops, $T L_{I}$, can be estimated by

$$
\begin{aligned}
T L_{I} & \leq \sum_{j=1}^{l}\left(2 B_{j}-3\right)\left|Q_{j-1}\right| \leq 2 \sum_{j=1}^{l}\left(B_{j}-1\right)\left|Q_{j-1}\right| \\
& \leq 2 \sum_{j=1}^{l}\left(B_{j}-1\right) \prod_{i=1}^{j-1} B_{i} \leq 2\left(\prod_{j=1}^{l} B_{j}-1\right) \leq 2 T-2 .
\end{aligned}
$$

For the maximum total number of multiplications in the inner while loops, $M_{I}$, we get

$$
\begin{aligned}
M_{I} & \leq 2 T-2+2 \sum_{j=1}^{l}\left(B_{j}-1\right)-2 l+\sum_{j=1}^{l}\left\lceil\log b_{j j}\right\rceil \\
& \leq 2 T+2 l(S) \cdot \sqrt{|G|}+\log |G|+l(S)-2 l-2,
\end{aligned}
$$

where we use that $\sum_{j=1}^{l}\left\lceil\log b_{j j}\right\rceil \leq \log \prod_{j=1}^{l} b_{j j}+l(S)=\log |G|+l(S)$.

Summing up, Algorithm 4.1 performs $l$ inversions, at most $(l+5) T+4 l(S) \sqrt{|G|}+$ $\log |G|$ group multiplications, and at most $(l+l(S)+2) T-2$ table look-ups. It requires space for at most $4 T$ pairs $(e, \vec{q}) \in G \times\{0, \ldots,|G|\}^{l}$. Since $T \leq 2^{\frac{l(S)}{2}} \sqrt{|G|}$ (see Lemma 4.2), this completes the proof.

To get reasonable lower bounds for the total number of group multiplications and table look-ups we should treat many different distributions and orders of $\left\{b_{11}, \ldots, b_{l l}\right\}$ separately. So we just give lower bounds for the sizes of $Q, R$ and $R^{\prime}$, and the number of group multiplications required to compute these sets. This is done in the proof of the following theorem.

Theorem 4.4. Let $\vec{v}=(2, \ldots, 2)$ in Algorithm 4.1. Then the number of group multiplications and the table sizes are 


$$
\Omega\left(\prod_{j=1}^{l}\left\lceil\sqrt{b_{j j}}\right\rceil\right),
$$

where $b_{11}, \ldots, b_{l l}$ denote the diagonal elements of the basis $\mathcal{B}$ computed by the algorithm.

Proof. Just as in the previous proof, let $Q_{j}, R_{j}$ and $R_{j}^{\prime}$ denote the sets $Q, R$ and $R^{\prime}$ after the $j$-th outer loop.

From the way $Q$ is built up we get that $\left|Q_{j}\right|=\left|Q_{j-1}\right|$ if $b_{j j}=1$, and $\left|Q_{j}\right| \geq$ $\left|Q_{j-1}\right|\left(B_{j}-1\right)$ if $B_{j}>1$ (i.e., if $\left.g_{j} \in E\right)$. Thus $\left|Q_{j}\right| \geq \prod_{\substack{i=1 \\ g_{i} \in E}}^{j}\left(B_{i}-1\right)$, so at the end of the algorithm we have

$$
|Q| \geq \prod_{\substack{j=1 \\ g_{j} \in E}}^{l-1}\left(B_{j}-1\right)=: \quad L,
$$

and it takes at least $L-1$ multiplications to compute all the sets $Q_{1}, \ldots, Q_{l-1}$. This lower bound is sharp: If $b_{j j} \leq 2$ for all $j$, then $Q=\{(1, \overrightarrow{0})\}$ at the end of the algorithm.

From the way $R$ is built up and from Theorem 2.3 we see that $\left|R_{j}\right|=0$ if $b_{j j}=1$, $\left|R_{j}\right| \geq\left|R_{j-1}^{\prime}\right|$ if $b_{j j}=2$, and $\left|R_{j}\right| \geq B_{j}\left|R_{j-1}^{\prime}\right|$ if $b_{j j} \geq 3$. With (10) it follows that

$$
\left|R_{j}\right| \geq \frac{1}{2} \prod_{\substack{i=1 \\ g_{j} \in E}}^{j} B_{i} .
$$

Let $p=\max \left\{j \in\{1, \ldots, l\}: g_{j} \in E\right\}$. Then $\left|R_{p}\right| \geq 1 / 2 \cdot T$, and it takes at least $1 / 2 \cdot T$ multiplications to compute $R_{p}$, with $T$ as in (9). It also follows from (10) that at the end of the algorithm we have that $\left|R^{\prime}\right|=T / B_{l}$. (Especially, $\left|R^{\prime}\right|=T$ if $g_{l} \notin E$.) This proves the theorem.

Corollary 4.5. Let $G=(\mathbb{Z} / 2 \mathbb{Z})^{k}$. Then Algorithm 4.1 is of complexity $\Omega(|G|)$.

Proof. Let $S$ be a generating sequence of $G$. Let $l=|S|$. Then for the diagonal elements $b_{j j}$ computed by the algorithm we necessarily have $k$ times $b_{j j}=2$ and $l-k$ times $b_{j j}=1$. Since $k=\log |G|$, the assertion follows by Lemma 4.2 together with Theorem 4.4.

Let us further comment on the factor $2^{\frac{l(S)}{2}}$, which appears in Theorem 4.3. We conclude from Theorem 4.4 that the more cyclic subgroups $G$ has and the smaller they are, the larger $R$ and $R^{\prime}$ (and $Q$ ) are, and thus the storage required and the number of group multiplications increases. However, this effect only depends on the structure of the type of groups we are dealing with. For example, in the case of groups of points on elliptic curves over finite fields, which are either cyclic or isomorphic to a product of two cyclic groups, this phenomenon is not relevant. In the case of ideal class groups of imaginary quadratic fields, where we expect small ranks [5], we can say that the worst case does not occur very frequently, especially for large discriminants.

Theoretically there is another possibility to have a disturbingly large exponent $l(S)$ even if $G$ is cyclic or consists of very large cyclic subgroups, namely if many generators are needed to build up each cyclic factor. 
To estimate the damage caused by these effects, we did the following experiments. For $n=3,4, \ldots, 10$ we took the first 1000 discriminants smaller than $10^{n}$. For each of these discriminants, we took the ten prime ideal classes of smallest norm in the corresponding imaginary quadratic order and used our algorithm to compute the subgroup generated by these classes. We measured the sizes of $Q$ and $R^{\prime}$ and counted the number $l(S)$ of prime ideal classes actually used in the algorithm to compute the subgroup. In Table 7 we compare $|Q|$ and $\left|R^{\prime}\right|$ with $|G|$ and $\sqrt{|G|}$, and in Table 8 we compare $l(S)$ with the number of cyclic factors of $|G|$, which is the minimum number of generators needed to compute $|G|$.

TABLE 7. Subgroup algorithm - size of $R^{\prime}$ and $Q$ relative to $|G|$ and $|G|^{1 / 2}$

\begin{tabular}{|r|c|c|c|c|c|c|c|c|}
\hline & \multicolumn{2}{|c|}{$|Q| /|G|$} & \multicolumn{2}{c|}{$\left|R^{\prime}\right| /|G|$} & \multicolumn{2}{c|}{$|Q| / \sqrt{|G|}$} & \multicolumn{2}{c|}{$\left|R^{\prime}\right| / \sqrt{|G|}$} \\
$n$ & $\max$ & ave & $\max$ & ave & $\max$ & ave & $\max$ & ave \\
\hline 3 & 0.50000 & 0.18271 & 1.00000 & 0.42068 & 1.30931 & 0.74079 & 5.65685 & 1.67457 \\
4 & 0.44444 & 0.11417 & 1.00000 & 0.28418 & 1.33333 & 0.71759 & 5.65685 & 1.74131 \\
5 & 0.19048 & 0.06389 & 0.66667 & 0.16840 & 1.25988 & 0.69884 & 5.36656 & 1.80439 \\
6 & 0.13187 & 0.03534 & 0.66667 & 0.09723 & 1.35710 & 0.68240 & 9.23760 & 1.83296 \\
7 & 0.06028 & 0.01932 & 0.26667 & 0.05590 & 1.34739 & 0.66498 & 7.15542 & 1.89770 \\
8 & 0.03896 & 0.01055 & 0.16000 & 0.03205 & 1.26179 & 0.64524 & 9.05097 & 1.93323 \\
9 & 0.01755 & 0.00581 & 0.09231 & 0.01835 & 1.31979 & 0.63294 & 8.41976 & 1.96326 \\
10 & 0.01094 & 0.00317 & 0.05424 & 0.01059 & 1.15923 & 0.61567 & 8.21715 & 2.02498 \\
\hline
\end{tabular}

TABLE 8. Subgroup algorithm — worst case data

\begin{tabular}{|r|c|c|c|c|}
\hline & \multicolumn{4}{|c|}{$l(S)$ number of cyclic factors } \\
$n$ & 0 & 1 & 2 & 3 \\
\hline 3 & 549 & 423 & 28 & 0 \\
4 & 520 & 422 & 58 & 0 \\
5 & 505 & 415 & 77 & 3 \\
6 & 525 & 385 & 84 & 6 \\
7 & 505 & 402 & 86 & 7 \\
8 & 537 & 380 & 77 & 6 \\
9 & 552 & 373 & 66 & 9 \\
10 & 536 & 384 & 75 & 5 \\
\hline
\end{tabular}

Table 7 shows that usually the sets $Q$ and $R^{\prime}$ do not blow up to the entire group size but contain less than $2 \sqrt{|G|}$ pairs $(g, \vec{q})$ in average. Only for discriminants with absolute value smaller than $10^{5}$ it occurred that $\left|R^{\prime}\right| \geq|G|$. Due to the way $Q$ is built up by Algorithm 4.1, the set $Q$ always contains considerably fewer elements than $R^{\prime}$.

Table 8 shows that in more than half of all our experiments the algorithm actually uses no more generators than theoretically necessary. Also in the remaining cases, $l(S)$ is always very small.

In Tables 9 and 10 we give some examples of subgroups computed with our algorithm. For each discriminant, we compute the group $G$ generated by the classes of the 10 prime ideals of smallest norms in the order. As before, $G M$ is the number 
of group multiplications required and $T L$ is the number of table look-ups. We used $\vec{v}=(2, \ldots, 2)$ as an initial step-width in all cases.

TABLE 9. Subgroup algorithm — sample run times

\begin{tabular}{|c|r|r|r|r|}
\hline$\Delta$ & $G$ & $G M$ & \multicolumn{1}{|c|}{$T L$} & \multicolumn{1}{c|}{ time } \\
\hline$-\left(10^{2}+3\right)$ & {$[5]$} & 50 & 14 & $0.03 \mathrm{sec}$ \\
$-\left(10^{3}+3\right)$ & {$[4]$} & 44 & 13 & $0.04 \mathrm{sec}$ \\
$-\left(10^{4}+3\right)$ & {$[12]$} & 59 & 17 & $0.05 \mathrm{sec}$ \\
$-\left(10^{5}+3\right)$ & {$[39]$} & 92 & 31 & $0.06 \mathrm{sec}$ \\
$-\left(10^{6}+3\right)$ & {$[105]$} & 122 & 51 & $0.08 \mathrm{sec}$ \\
$-\left(10^{7}+3\right)$ & {$[706]$} & 332 & 149 & $0.20 \mathrm{sec}$ \\
$-\left(10^{8}+3\right)$ & {$[1702]$} & 421 & 196 & $0.28 \mathrm{sec}$ \\
$-\left(10^{9}+3\right)$ & {$[1840,2]$} & 595 & 245 & $0.40 \mathrm{sec}$ \\
$-\left(10^{10}+3\right)$ & {$[10538]$} & 1038 & 369 & $0.73 \mathrm{sec}$ \\
$-\left(10^{11}+3\right)$ & {$[31057]$} & 2213 & 1067 & $1.60 \mathrm{sec}$ \\
$-\left(10^{12}+3\right)$ & {$[62284,2]$} & 3223 & 1989 & $2.56 \mathrm{sec}$ \\
$-\left(10^{13}+3\right)$ & {$[124264,2,2]$} & 5794 & 2464 & $4.25 \mathrm{sec}$ \\
$-\left(10^{14}+3\right)$ & {$[356368,2,2]$} & 9233 & 3751 & $7.53 \mathrm{sec}$ \\
$-\left(10^{15}+3\right)$ & {$[3929262]$} & 23564 & 13182 & $18.41 \mathrm{sec}$ \\
$-\left(10^{16}+3\right)$ & {$[12284352]$} & 37249 & 16409 & $30.36 \mathrm{sec}$ \\
$-\left(10^{17}+3\right)$ & {$[38545929]$} & 67130 & 29484 & $1 \mathrm{~min}, 0.12 \mathrm{sec}$ \\
$-\left(10^{18}+3\right)$ & {$[102764373]$} & 103039 & 54913 & $1 \mathrm{~min}, 30.62 \mathrm{sec}$ \\
$-\left(10^{19}+3\right)$ & {$[78425040,2,2,2]$} & 149197 & 83049 & $3 \mathrm{~min}, 39.47 \mathrm{sec}$ \\
$-\left(10^{20}+3\right)$ & {$[721166712,2]$} & 343423 & 210837 & $6 \mathrm{~min}, 13.87 \mathrm{sec}$ \\
\hline
\end{tabular}

TABLE 10. Subgroup algorithm - sample run times

\begin{tabular}{|c|r|r|r|r|}
\hline$\Delta$ & \multicolumn{1}{|c|}{$G$} & \multicolumn{1}{|c|}{$G M$} & \multicolumn{1}{|c|}{$T L$} & \multicolumn{1}{c|}{ time } \\
\hline$-4\left(10^{2}+1\right)$ & {$[14]$} & 63 & 18 & $0.03 \mathrm{sec}$ \\
$-4\left(10^{3}+1\right)$ & {$[10,2,2]$} & 87 & 30 & $0.04 \mathrm{sec}$ \\
$-4\left(10^{4}+1\right)$ & {$[40,4]$} & 140 & 58 & $0.06 \mathrm{sec}$ \\
$-4\left(10^{5}+1\right)$ & {$[230,2]$} & 223 & 91 & $0.10 \mathrm{sec}$ \\
$-4\left(10^{6}+1\right)$ & {$[516,2]$} & 319 & 114 & $0.13 \mathrm{sec}$ \\
$-4\left(10^{7}+1\right)$ & {$[1446,2]$} & 598 & 243 & $0.26 \mathrm{sec}$ \\
$-4\left(10^{8}+1\right)$ & {$[4104,4]$} & 1223 & 640 & $0.71 \mathrm{sec}$ \\
$-4\left(10^{9}+1\right)$ & {$[2560,2,2,2,2]$} & 1509 & 385 & $0.80 \mathrm{sec}$ \\
$-4\left(10^{10}+1\right)$ & {$[48396,2,2]$} & 3388 & 1622 & $1.93 \mathrm{sec}$ \\
$-4\left(10^{11}+1\right)$ & {$[56772,2,2,2]$} & 4891 & 2660 & $2.54 \mathrm{sec}$ \\
$-4\left(10^{12}+1\right)$ & {$[117360,4,2]$} & 6680 & 3323 & $3.76 \mathrm{sec}$ \\
$-4\left(10^{13}+1\right)$ & {$[742228,2,2]$} & 12037 & 4233 & $7.11 \mathrm{sec}$ \\
$-4\left(10^{14}+1\right)$ & {$[1159048,4,2,2]$} & 27615 & 11729 & $16.91 \mathrm{sec}$ \\
$-4\left(10^{15}+1\right)$ & {$[257448,4,2,2,2,2,2]$} & 57387 & 22013 & $36.58 \mathrm{sec}$ \\
$-4\left(10^{16}+1\right)$ & {$[11809616,2,2,2,2]$} & 120027 & 26425 & $1 \mathrm{~min}, 11.94 \mathrm{sec}$ \\
$-4\left(10^{17}+1\right)$ & {$[46854696,2,2,2]$} & 134990 & 65584 & $1 \mathrm{~min}, 28.21 \mathrm{sec}$ \\
$-4\left(10^{18}+1\right)$ & {$[264135076,2,2]$} & 233224 & 94688 & $2 \mathrm{~min}, 40.54 \mathrm{sec}$ \\
$-4\left(10^{19}+1\right)$ & {$[1649441906,2]$} & 572162 & 236334 & $6 \mathrm{~min}, 33.53 \mathrm{sec}$ \\
$-4\left(10^{20}+1\right)$ & {$[1856197104,2,2,2]$} & 979126 & 380022 & $15 \mathrm{~min}, 39.46 \mathrm{sec}$ \\
\hline
\end{tabular}




\section{REFERENCES}

[1] I. Biehl and J. Buchmann, Algorithms for quadratic orders, Proc. Sympos. Appl. Math., vol. 48, Amer. Math. Soc., Providence, RI, 1994. MR 95m:11146

[2] J. Buchmann and S. Paulus, Algorithms for finite abelian groups, Extended abstract. To be published in the proceedings of NTAMCS 93.

[3] D.A. Buell, Binary quadratic forms: classical theory and modern computations, SpringerVerlag, New York, 1989. MR 92b:11021

[4] H. Cohen, A course in computational algebraic number theory, Springer-Verlag, Berlin, 1993. MR 94i: 11105

[5] H. Cohen and H.W. Lenstra, Jr., Heuristics on class groups of number fields, Number Theory, Lecture Notes in Math., vol. 1068, Springer-Verlag, New York, 1984, pp. 33-62. MR 85j:11144

[6] P.D. Domich, Residual Hermite normal form computations, ACM Transactions on Mathematical Software 15 (1989), no. 3, 275-286. MR 91d:15020

[7] J. Buchmann I. Biehl and T. Papanikolaou, LiDIA - a library for computational number theory, The LiDIA Group, Universität des Saarlandes, Saarbrücken, Germany, 1995.

[8] A.K. Lenstra and H.W. Lenstra, Jr., Algorithms in number theory, Handbook of theoretical computer science (J. van Leeuwen, ed.), Elsevier Science Publishers, 1990, pp. 673-715. CMP 92:01

[9] S. Paulus, Algorithmen für endliche abelsche Gruppen, Master's thesis, Universität des Saarlandes, Saarbrücken, Germany, 1992.

[10] D. Shanks, Class number, a theory of factorization and genera, Proc. Symp. Pure Math. 20, AMS, Providence, R.I., 1971, pp. 415-440. MR 47:4932

Technische Hochschule Darmstadt, Institut für Theoretische Informatik, AlexanDerstrasse 10,64283 Darmstadt, Germany

E-mail address, J. Buchmann: buchmann@cdc.informatik.th-darmstadt.de

E-mail address, M. J. Jacobson, Jr.: jacobs@cdc.informatik.th-darmstadt.de

E-mail address, E. Teske: teske@cdc.informatik.th-darmstadt.de 